\title{
Regional Anesthesia Facilitates the Early Recognition of Local Anesthetic Toxicity
}

\author{
Emmanuel Alalade ${ }^{\mathrm{a}, \mathrm{d}}$, Lance M. Relland ${ }^{\mathrm{a}, \mathrm{b}}$, Kristin Chenault ${ }^{\mathrm{a}, \mathrm{b}}$, Nicole Elsey ${ }^{\mathrm{a}, \mathrm{b}}$, \\ Molly Fuchs ${ }^{\mathrm{c}}$, Seth Alpert ${ }^{\mathrm{c}}$, Joseph Tobias ${ }^{\mathrm{a}, \mathrm{b}}$
}

\begin{abstract}
Rising concerns regarding the potential long-term neurocognitive effects of general anesthetic agents have renewed an interest in using regional anesthesia instead of general anesthesia in infants. Although generally safe and effective, the primary risk associated with regional anesthesia relates to the use of large doses of local anesthetic agents and the potential for local anesthetic systemic toxicity (LAST). We present three infants who suffered LAST after receiving regional anesthesia instead of general anesthesia. The early signs and symptoms were quickly identified in the awake state thereby allowing for cessation of continuous drug administration and a rapid response to treat LAST before progression to severe sequelae.
\end{abstract}

Keywords: Spinal anesthesia; Local anesthesia systemic toxicity; Regional anesthesia

\section{Introduction}

Regional anesthesia is frequently combined with general anesthesia in the pediatric-aged patient to provide postoperative analgesia and avoid the need for systemic opioids [1]. Given recent concerns regarding the effects of general anesthetic agents on long-term neurocognitive outcomes, regional anesthesia (spinal anesthesia or combined spinal/caudal epidural anesthesia) is also being offered as an alternative to general anesthesia [2-4]. There was a rigorous period of preparation prior to the public offering of the program. Pediatric anesthesiologists with regional experiences were able to be proficient

Manuscript submitted November 22, 2019, accepted November 29, 2019

aDepartment of Anesthesiology and Pain Medicine, Nationwide Children's Hospital, Columbus, OH, USA

${ }^{b}$ Department of Anesthesiology and Pain Medicine, The Ohio State University College of Medicine, Columbus, OH, USA

'Department of Pediatric Urology, Nationwide Children's Hospital, Columbus, $\mathrm{OH}$, USA

${ }^{\mathrm{d} C}$ Corresponding Author: Emmanuel Alalade, Department of Anesthesiology and Pain Medicine, Nationwide Children's Hospital, 700 Children's Drive, Columbus, OH 43205, USA.

Email: Emmanuel.Alalade@Nationwidechildrens.org

doi: https://doi.org/10.14740/jmc3393 with spinal anesthesia in five procedures or less. However, the successful system-wide integration for the whole pediatric anesthesia faculty was approximately 6 months with proficiency achieved on average by the 10th attempt. While generally safe and effective, adverse effects may occur during regional anesthesia in infants and children, the most significant being local anesthetic systemic toxicity (LAST) [5]. Unlike the adult population, both neuraxial and peripheral nerve blockade are typically performed under deep sedation or general anesthesia in infants and children such that signs and symptoms of LAST may not detectable until the later stages of cardiovascular collapse [6]. Techniques to decrease the incidence of LAST include careful dose calculation, slow incremental injection with intermittent aspiration, use of ultrasound guidance, and a test dose containing epinephrine to identify inadvertent systemic administration [7-9].

Procedures performed using regional anesthesia (spinal or combined spinal/caudal anesthesia) may enable patients to remain awake or lightly sedated during surgical procedures, which could facilitate the early recognition of LAST through the identification of central nervous system (CNS) effects that conventionally precede cardiotoxicity. We recently cared for three infants who each exhibited early signs of LAST while awake during surgical procedures performed under regional anesthesia. We review the mechanisms, presentation, risk factors and management of LAST in infants and children. Although multiple studies have supported the safety of regional techniques under general anesthesia in infants and children, we discuss the theoretical benefit of having an awake patient while performing regional anesthesia even in the pediatric-aged patient, as this may allow the manifestation of CNS signs and symptoms of LAST prior to cardiovascular toxicity $[5,10]$.

\section{Case Reports}

The retrospective review of these cases and presentation in this format was approved by the Institutional Review Board at Nationwide Children's Hospital (Columbus, OH, USA). In all three patients, the technical approach for performance of spinal anesthesia was similar. All patients received a topical anesthetic cream (LMX (4\% lidocaine)) that was applied over the lumbar region in the perioperative area after informed consent was obtained. The patients were transferred to the operating room and standard American Society of Anesthesiologists' monitors were placed. The topical cream was removed, and 
the lumbar area cleaned with chlorhexidine. With the patient in the sitting position, the spinal anesthetic was administered in a sterile fashion using a styletted 22-gauge, 1.5-inch spinal needle at the L4 - L5 interspace. After free flow of cerebrospinal fluid was obtained, isobaric bupivacaine $(0.5 \%)$ containing 1:200,000 epinephrine and clonidine was injected. The infants were then placed in the supine position and a peripheral intravenous catheter was placed in the lower extremity. Adequate motor and sensory blockade was confirmed prior to the start of the surgical procedure.

\section{Case 1}

A 3-month-old, 49-week post-conceptual age (PCA), $8.3 \mathrm{~kg}$ male presented for correction of congenital chordee. The intrathecal anesthetic in this case was $1.2 \mathrm{~mL}$ of isobaric $0.5 \%$ bupivacaine containing 1:200,000 epinephrine and $8 \mu \mathrm{g}$ of clonidine. No significant change in hemodynamic status was noted after placement of spinal anesthesia. Upon completion of the surgical procedure, the surgeon administered a dorsal penile block using $4 \mathrm{~mL}$ of $0.25 \%$ bupivacaine to provide postoperative analgesia. Immediately after this injection, the patient exhibited jerking/twitching movements, a heart rate increase $\geq 30$ beats/min, and a decreased oxygen desaturation. Assisted-ventilation with a bag-valve-mask, $100 \%$ oxygen and the subsequent administration of propofol $(20 \mathrm{mg})$ and midazolam $(0.5 \mathrm{mg})$ resulted in cessation of the seizure activity. The patient remained hemodynamically stable and did not require endotracheal intubation. He was transported to the post-anesthesia care unit (PACU) and monitored for $2 \mathrm{~h}$ with no reoccurrence of the seizure activity. The patient was discharged home and had no postoperative issues.

\section{Case 2}

A 7-month-old, 8.6-kg male presented for circumcision, scrotoplasty and hidden penis repair. Spinal anesthesia was induced using of $1.1 \mathrm{~mL}$ of isobaric $0.5 \%$ bupivacaine containing 1:200,000 epinephrine and $9 \mu \mathrm{g}$ of clonidine. Sixty-five minutes later, at the end of the surgical procedure, the surgeon placed a dorsal penile block by injecting $5 \mathrm{~mL}$ of $0.25 \%$ bupivacaine. Immediately thereafter, the patient cried out and started to have rhythmic jerking movements of the head, eyes and upper extremities, with frothing from the mouth. He subsequently became apneic and tachycardic. This patient was similarly treated with $100 \%$ oxygen, propofol $(5 \mathrm{mg})$ and midazolam $(0.25 \mathrm{mg})$ which resulted quickly in complete cessation of the seizure activity. The patient was transported to PACU in stable condition with no focal neurological deficits. He was discharged home later in the day. His postoperative course was unremarkable.

\section{Case 3}

A 7-month-old, 8.2-kg male presented for first stage hypospa- dias repair. The intrathecal administration of $1.2 \mathrm{~mL}$ of isobaric $0.5 \%$ bupivacaine containing 1:200,000 epinephrine, and $8 \mu \mathrm{g}$ of clonidine was followed by placement of a caudal epidural catheter that was tested with negative aspiration and a test dose. During the case, the patient was initially awake, interactive with a heart rate trending down from 140 to 100 beats/min before falling asleep. An hour after placement of the spinal anesthesia, $12 \mathrm{~mL}$ of $3 \%$ chloroprocaine $(360 \mathrm{mg}$ ) was injected through the caudal catheter before and an infusion started at $1 \mathrm{~mL} / \mathrm{kg} / \mathrm{h}$ to maintain an appropriate level of anesthesia. Twenty minutes later, a notable oxygen desaturation was accompanied by upper body twitching and a heart rate increase $\geq 20$ beats $/ \mathrm{min}$. The epidural infusion was immediately suspended, and ventilation was supported with $100 \%$ oxygen via bag-valve-mask ventilation. The twitching ceased after the administration of midazolam (1 mg). Surgery was completed approximately $15 \mathrm{~min}$ after stopping the infusion of chloroprocaine, and no further local anesthetic agent was administered through the caudal catheter. The patient was discharged from the PACU after he was fully awake with a normal neurological exam. He was discharged home that day with no sequelae from the seizure activity. His postoperative course was uneventful. The patient subsequently had the second stage of the procedure under regional anesthesia without any adverse event.

\section{Discussion}

Spinal anesthesia is offered commonly as an alternative to general anesthesia in neonates and infants to avoid the possible adverse neurocognitive effects of general anesthetic agents. Clinical experience has demonstrated that spinal anesthesia is a safe alternative to general anesthesia for infra-umbilical surgical procedures lasting less than $70-80 \mathrm{~min}$ [11]. It requires a minimal amount of the local anesthetic agent and is associated with low blood concentrations of the local anesthetic agent. In our first two patients, as the duration of surgical anesthesia is $70-80 \mathrm{~min}$, an additional technique such as a penile nerve block is required to provide prolonged postoperative analgesia. In our third patient, a combined spinal-epidural technique was chosen as the anticipated duration of the surgical procedures was longer than that provided by a single shot spinal anesthetic technique $[3,4]$.

In addition to avoiding the potential neurocognitive effects of general anesthetic agents, regional anesthesia supports hemodynamic stability, is associated with lower intraoperative blood loss, and blunts the surgical stress-hormone response [1]. However, as with any anesthetic technique, adverse effects may occur with regional anesthesia. This may include injuries related to needle placement or reactions to the subsequent administration of the local anesthetic agent. Systemic toxicity from local anesthetic administration may occur due to the administration of a large single dose, inadvertent systemic injection, rapid systemic absorption, redosing or a continuous infusion [12]. LAST generally affects the central nervous and the cardiovascular systems with the former generally appearing first and at lower blood concentrations [13].

The earliest signs of systemic toxicity result from the 
blockade of inhibitory pathways in the cerebral cortex [14]. This results in secondary excitatory nerve hyperactivity leading to shivering, myoclonus, tremors and sudden muscular contractions with progression to tonic-clonic seizures [15]. These signs and symptoms of CNS hyperactivity generally occur at a lower plasma concentration, preceding cardiotoxicity and cardiovascular collapse [16]. Arrhythmias and myocardial depression are the two forms of cardiotoxicity. The gamut of arrhythmias varies from tachycardia to asystole. There is a disruption of the intrinsic conduction system via the blockade of the sodium and potassium channels [17]. The structure of the sodium channels closely mirrors the voltage-dependent calcium channels; hence local anesthetic agents block these channels by the same mechanism [18]. The effects on the calcium channels decreases contractility by inhibiting intracellular calcium release from the sarcoplasmic reticulum. In addition, there is a significant reduction in the generation of cyclic adenosine monophosphate (AMP), which results from the inactivation of $\beta$-adrenergic receptors and depressed adenylate cyclase activity [19].

Given the issues with reversing the cardiovascular effects of LAST, early recognition prior to the onset of cardiovascular toxicity may be beneficial. However, in the pediatric-aged patient, regional anesthesia is generally used in association with general anesthesia with or without neuromuscular blockade. Therefore, the early CNS signs and symptoms of LAST may not be appreciated such that the first manifestation of an accidental systemic injection or rapid bolus may be cardiovascular collapse $[20,21]$. Furthermore, general anesthesia may also adversely affect the pharmacokinetics and distribution of local anesthetic agents thereby increasing the potential for systemic toxicity $[22,23]$.

Our three cases demonstrate the potential benefit of avoiding the concomitant use of general anesthesia with regional anesthesia. Given the location of the surgical procedure, these cases are easily amenable to a regional anesthetic technique. Nevertheless, as opposed to the routine practice in the adult population, the convention for pediatric patients is to frequently provide a general anesthetic and use the regional only as an adjunctive technique. Our experience has clearly demonstrated that infants and children are able to tolerate regional anesthesia without general anesthesia and frequently without sedation. As our three patients were not anesthetized, sedated or receiving a neuromuscular blocking agent, LAST was identified early by a seizure. The propagation of the seizure activity immediately ceased after intervention, which we postulate prevented progression to cardiovascular decompensation by maintaining metabolic homeostasis with early airway intervention or early cessation of the infusion. Prevention of acidosis and hypoxia with adequate seizure control and airway management in the setting of LAST has been shown to prevent the progression to cardiovascular collapse [24]. In our third patient, the onset of seizure activity not only alerted us to the presence of LAST for an immediate termination of the infusion, but also allowed recognition of a program error that involved the administration of 1.5 times the appropriate amount of chloroprocaine. This resulted in immediate treatment of the seizure and discontinuation of the chloroprocaine infusion through the caudal epidural catheter before the onset of cardiovascular toxicity.
In addition, our practice for continuous epidural infusions in infants and children less than 1 year of age routinely includes the use of chloroprocaine $[25,26]$. If an amide local anesthetic of the amide class (bupivacaine or ropivacaine) had been used, there would likely be a higher risk of cardiovascular collapse. Chloroprocaine is rapidly metabolized and only transient toxic effects have been reported for a similar clinical scenario [27].

In the most recent analysis from the Pediatric Regional Anesthesia Network of more than 100,000 blocks, the incidence of local anesthetic toxicity was 0.76 per 10,000 with the majority of cases occurring in infants [5]. Severe LAST occurred in seven cases, five of which were infants. The cases all included the use of regional anesthesia with general anesthesia or sedation and presented as cardiovascular collapse $(n=4)$ or seizures $(n=3)$ with some requiring the initiation of intralipid and inotropic support. These data demonstrate that infants less than 6 months are at higher risk for LAST, and that it is often not a consequence of excessive dosing, but rather other factors such as unrecognized systemic injection, rapid absorption and distribution, or decreased hepatic $\alpha_{1}$-acid glycoprotein synthesis in young infants [5]. Our case series support these findings, and show the benefit of having an awake patient under regional anesthesia to allow for early recognition of LAST, discontinuation of an ongoing infusion, and appropriate intervention to prevent hypoxia or acidosis prior to possible progression to cardiovascular collapse. However, should cardiovascular toxicity occur, rapid treatment using a standard intralipid is recommended [7].

Excessive dosing was the most likely cause of seizure in our third reported case. In the former two cases, despite a careful administration technique that elicited appropriate negative aspiration, an apparent inadvertent systemic injection occurred during the dorsal penile nerve blocks, demonstrating that even with appropriate technique and dose calculation, LAST can occur if systemic administration is rapid. With increased awareness of LAST and use of techniques to prevent its occurrence, the overall incidence of LAST seems to be decreasing. However, this may not be the case with specific blocks such as the dorsal penile nerve block, where rapid absorption of local anesthetic agents may occur [28]. With the overall decrease in the number of LAST cases, those related to dorsal penile nerve blocks may be proportionally increasing, leading some practitioners to suggest that a lower dose of bupivacaine (1.25 $\mathrm{mg} / \mathrm{kg}$ ) should be used [28].

Despite the episodes described in our three patients, they did not have an increase in their expected length of stay, and they were discharged home the same day after observation in the PACU. No long-term sequelae were noted on follow-up visits. The high safety profile for performing spinal anesthesia in awake pediatric patients is enhanced through early detection of LAST, which permits expeditious intervention, immediate treatment and maintenance of homeostasis to prevent possible progression to cardiovascular collapse.

\section{Acknowledgments}

None to declare. 


\section{Financial Disclosure}

The authors have no financial disclosure to declare.

\section{Conflict of Interest}

None to declare.

\section{Informed Consent}

Informed consent was obtained.

\section{Author Contributions}

Emmanuel Alalade MD is the first author involved in the literature review and preparation of the manuscript (initial and revised versions). EA, LR, KC, NE, MF, SA, and JT were involved in the critical revision of all versions of the manuscript.

\section{References}

1. Bosenberg A. Benefits of regional anesthesia in children. Paediatr Anaesth. 2012;22(1):10-18.

2. Whitaker EE, Wiemann BZ, DaJusta DG, Alpert SA, Ching CB, McLeod DJ, Tobias JD, et al. Spinal anesthesia for pediatric urological surgery: Reducing the theoretic neurotoxic effects of general anesthesia. J Pediatr Urol. 2017;13(4):396-400.

3. Trifa M, Tumin D, Whitaker EE, Bhalla T, Jayanthi VR, Tobias JD. Spinal anesthesia for surgery longer than 60 min in infants: experience from the first 2 years of a spinal anesthesia program. J Anesth. 2018;32(4):637-640.

4. Jayanthi VR, Spisak K, Smith AE, Martin DP, Ching CB, Bhalla T, Tobias JD, et al. Combined spinal/caudal catheter anesthesia: extending the boundaries of regional anesthesia for complex pediatric urological surgery. J Pediatr Urol. 2019;15(5):442-447.

5. Walker BJ, Long JB, Sathyamoorthy M, Birstler J, Wolf $\mathrm{C}$, Bosenberg AT, Flack $\mathrm{SH}$, et al. Complications in pediatric regional anesthesia: an analysis of more than 100,000 blocks from the pediatric regional anesthesia network. Anesthesiology. 2018;129(4):721-732.

6. Ivani G, Suresh S, Ecoffey C, Bosenberg A, Lonnqvist PA, Krane E, Veyckemans F, et al. The European Society of Regional Anaesthesia and Pain Therapy and the American Society of Regional Anesthesia and Pain Medicine Joint Committee Practice Advisory on controversial topics in pediatric regional anesthesia. Reg Anesth Pain Med. 2015;40(5):526-532.

7. Neal JM, Barrington MJ, Fettiplace MR, Gitman M, Memtsoudis SG, Morwald EE, Rubin DS, et al. The Third American Society of Regional Anesthesia and Pain Medicine Practice Advisory on local anesthetic systemic toxicity: executive summary 2017. Reg Anesth Pain Med.
2018;43(2):113-123.

8. Barrington MJ, Kluger R. Ultrasound guidance reduces the risk of local anesthetic systemic toxicity following peripheral nerve blockade. Reg Anesth Pain Med. 2013;38(4):289-299.

9. Tobias JD. Caudal epidural block: a review of test dosing and recognition of systemic injection in children. Anesth Analg. 2001;93(5):1156-1161.

10. Taenzer AH, Walker BJ, Bosenberg AT, Martin L, Suresh $\mathrm{S}$, Polaner DM, Wolf C, et al. Asleep versus awake: does it matter?: Pediatric regional block complications by patient state: a report from the Pediatric Regional Anesthesia Network. Reg Anesth Pain Med. 2014;39(4):279-283.

11. Tobias JD. Spinal anaesthesia in infants and children. Paediatr Anaesth. 2000;10(1):5-16.

12. Berde CB. Toxicity of local anesthetics in infants and children. J Pediatr. 1993;122(5 Pt 2):S14-20.

13. Dickerson DM, Apfelbaum JL. Local anesthetic systemic toxicity. Aesthet Surg J. 2014;34(7):1111-1119.

14. Scott DB. Toxic effects of local anaesthetic agents on the central nervous system. Br J Anaesth. 1986;58(7):732735 .

15. Scott DB. Evaluation of clinical tolerance of local anaesthetic agents. Br J Anaesth. 1975;47(suppl):328-331.

16. Mather LE, Tucker GT, Murphy TM, Stanton-Hicks MD, Bonica JJ. Cardiovascular and subjective central nervous system effects of long-acting local anaesthetics in man. Anaesth Intensive Care. 1979;7(3):215-221.

17. Groban L. Central nervous system and cardiac effects from long-acting amide local anesthetic toxicity in the intact animal model. Reg Anesth Pain Med. 2003;28(1):311.

18. Scholz A. Mechanisms of (local) anaesthetics on voltage-gated sodium and other ion channels. Br J Anaesth. 2002;89(1):52-61.

19. Stehr SN, Ziegeler JC, Pexa A, Oertel R, Deussen A, Koch $\mathrm{T}$, Hubler M. The effects of lipid infusion on myocardial function and bioenergetics in 1-bupivacaine toxicity in the isolated rat heart. Anesth Analg. 2007;104(1):186-192.

20. Dillane D, Finucane BT. Local anesthetic systemic toxicity. Can J Anaesth. 2010;57(4):368-380.

21. Ved SA, Pinosky M, Nicodemus H. Ventricular tachycardia and brief cardiovascular collapse in two infants after caudal anesthesia using a bupivacaine-epinephrine solution. Anesthesiology. 1993;79(5):1121-1123.

22. Di Gregorio G, Neal JM, Rosenquist RW, Weinberg GL. Clinical presentation of local anesthetic systemic toxicity: a review of published cases, 1979 to 2009. Reg Anesth Pain Med. 2010;35(2):181-187.

23. Copeland SE, Ladd LA, Gu XQ, Mather LE. The effects of general anesthesia on whole body and regional pharmacokinetics of local anesthetics at toxic doses. Anesth Analg. 2008;106(5):1440-1449, table of contents.

24. Moore DC, Crawford RD, Scurlock JE. Severe hypoxia and acidosis following local anesthetic-induced convulsions. Anesthesiology. 1980;53(3):259-260.

25. Veneziano G, Iliev P, Tripi J, Martin D, Aldrink J, Bhalla T, Tobias J. Continuous chloroprocaine infusion for thoracic and caudal epidurals as a postoperative analge- 
sia modality in neonates, infants, and children. Paediatr Anaesth. 2016;26(1):84-91.

26. Veneziano G, Tobias JD. Chloroprocaine for epidural anesthesia in infants and children. Paediatr Anaesth. 2017;27(6):581-590.

27. Hernandez MA, Boretsky K. Chloroprocaine: local anes- thetic systemic toxicity in a 9-month infant with paravertebral catheter. Paediatr Anaesth. 2016;26(6):665-666.

28. Yu RN, Houck CS, Casta A, Blum RH. Institutional policy changes to prevent cardiac toxicity associated with bupivacaine penile blockade in infants. A A Case Rep. 2016;7(3):71-75. 